\title{
The Hennepin Ketamine Study
}

\author{
Samuel J. Stratton, MD, MPH
}

An Emergency Medical Services (EMS) clinical trial in the United States has generated controversy that has an impact on the future of prospective design prehospital and disaster research throughout the world. The clinical trial utilized a method, termed in the US, as "Exception from Informed Consent for Emergency Research." ${ }^{1}$ This method allows for the prospective study of lifethreatening emergency conditions without immediate patient consent. Exception of informed consent is appropriate for studies of emergency life-threatening conditions in which consent is not feasible due to necessity for urgent intervention. The urgent intervention must be expected to have life-saving benefit but may have more than minimal health risks. The intervention would also be one that requires scientific evidence to determine safety and effectiveness. Another qualification for research under exception from informed consent is that study subjects cannot give consent because of their medical condition.

It is important not to confuse exception from informed consent with another research design termed "waiver" of informed consent. The primary difference between the two forms for research consent is whether there is risk to an individual from the research intervention. When a research proposal has no more than minimal risk, a human research ethics committee may waive some or all elements of informed consent. Waiver of informed consent is common in emergency and disaster research when the study is designed as an analysis of existing data, such as in medical record or medical database reviews.

In June of this year, the Minneapolis (Minnesota USA) Star Tribune newspaper reported that ketamine was administered by EMS personnel to individuals after police at the scene urged that the drug be given. ${ }^{2}$ At the time, ketamine was being evaluated in a prehospital clinical trial comparing the drug to midazolam for sedation of persons with agitated delirium. The study was conducted using exception of informed consent and was approved by the Hennepin Healthcare Human Subjects Research Protection Committee (called Institutional Review Board in the US; Minneapolis, Minnesota USA). The initial Star Tribune news story was picked up by numerous other media, including the New York Times (New York USA) ${ }^{3}$ and Forbes (New Jersey USA). ${ }^{4}$ The media were highly critical of Minneapolis police officers, but the issue was not brought to public attention until the Minneapolis Police Department had started and determined that going forward, police officers were not to suggest or demand that EMS personnel sedate a person. ${ }^{3}$ An underlying issue was the use of an anesthetic drug that causes seemingly strange dissociation reactions to control agitated delirium of individuals within politically vulnerable populations primarily represented by low-income, homeless, and minority groups.

With the spread of media coverage, the situation further evolved. Appropriately, Hennepin Healthcare suspended the prehospital ketamine study. Advocacy groups filed an ethics complaint with the US oversight agencies (Food and Drug Administration [Silver Spring, Maryland USA] and Office for Human Research Protections [Rockville, Maryland USA]) against those involved with the clinical trial. The media further portrayed the local community as being outraged by the study and conduct of EMS and police. Some political figures in the area began to take advantage to use public forums to express their "outrage" at the trial, obviously seeking public attention and future votes for political office. Media, non-medical sources, and "medical experts" described ketamine as a "powerful anesthetic" that represented a serious safety and health risk when administered to humans. Ironically in the US, ketamine is becoming accepted as an appropriate EMS drug without a well-designed and conducted prospective clinical trial. ${ }^{5}$

Particularly concerning is media and political comparisons of the Hennepin ketamine trial to the past serious "study" violations of human rights and life such as human experimentation during World War II and the US Tuskegee experiments of 1932 to 1972 in which untreated syphilis was observed for long-term effects without consent of the men who had the disease and there was a known effective treatment.

The Hennepin ketamine study was based on exemption from informed consent because the study was prospective and included patients whose medical condition (agitated delirium) precluded consent prior to therapy. There is sufficient literature to establish that agitated delirium is potentially life-threatening, making the study eligible for exemption from informed consent. In the US, as well as other nations, five elements must be met when a study is conducted with exemption from informed consent. These elements include community consultation for opinion and concern regarding the proposed study, public disclosure of the study before and after the trial, plans for obtaining consent from available authorized study subject representatives, formation of a data safety monitoring board with members that are not involved with the study, and filing an application for an Investigational New Drug with the US Food and Drug Administration.

The goal of community consultation and public disclosure is to discuss and obtain opinions regarding a study prior to initiating a study. The consultation and public disclosure are focused to the community from which study subjects are drawn. Consultation and public disclosure are not a substitution for individual consent and rather is a means for the community in which potential study subjects are drawn to become informed about the research and express views about it. Acceptable consultation and public disclosure require addressing community-level concerns for the research.

In reporting on the Hennepin ketamine study, Forbes listed a number of issues. ${ }^{4}$ While the Forbes issues are reported by a media outlet and not otherwise confirmed, they include: (1) The study was not randomized or blinded between the two drugs (ketamine and midazolam) allowing EMS personnel to know which medication was being administered; (2) It is unclear if the indications for entering someone in the study were standardized or validated (potential selection bias and over-treatment risk); and (3) Consent was not sought when a patient representative or surrogate was immediately available. 
Cultural and social competency are as important as the technical aspects of a clinical trial. The Hennepin ketamine study explored medical sedation in the setting of agitated delirium, a condition that is more likely to occur among low-income and minority groups as well as those with mental health challenges. These groups are considered vulnerable and less likely to have control of their personal rights and medical decisions. It is unclear if these groups were approached or capable of understanding the Hennepin ketamine study during the community consultation and public disclosure phases of setting up the trial. These groups are difficult for investigators to approach and often have mistrust of established institutions which leads to resistance for accepting initiatives such as clinical trials. In essence, respect and understanding the culture and fears of a community are of high

References

1. US Department of Health and Human Services, Food and Drug Administration. Guidance for Institutional Review Boards, clinical investigators, and sponsors: exception from informed consent requirements for emergency research. Published April 2013.

2. Mannix A. At urging of Minneapolis police, Hennepin EMS workers subdued dozens with a powerful sedative. Minneapolis Star Tribune. Published June 15, 2018.

3. Mele C. Ketamine used to subdue dozens at request of Minneapolis Police, report says. The New York Times. Published June 16, 2018. importance when conducting research using exception from informed consent.

To accomplish full status as a scientific discipline, emergency and disaster research requires prospective, comparative research to develop valid science for advancement. Without clinical trials, emergency and disaster medicine is denigrated to opinion and bias as the basis for practice. The Hennepin ketamine studies will result in tighter scrutiny of exempt from consent trials. While this tighter supervision by researchers and human research committees will possibly address community distrust of emergency research, it will result in more difficulty in conduct of trials. Above all, emergency and disaster research is community-based and requires cultural competency, supervision of field personnel, and adherence to established research ethics.

4. Stone J. Hennepin ketamine study raises questions about ethics and consent. Forbes. Published July 26, 2018.

5. Buckland DM, Crowe R, Cash RE, et al. Ketamine in the prehospital environment: a national survey of paramedics in the United States. Prehosp Disaster Med. 2018;33 (1):23-28.

doi:10.1017/S1049023X18000924 